\title{
Transverse Radiation realized as Deformed Harmonic Oscillators
}

\author{
P. Narayana Swamy \\ Department of Physics, Southern Illinois University, Edwardsville IL 62026
}

\begin{abstract}
We present a theory of quantized radiation fields described in terms of qdeformed harmonic oscillators. The creation and annihilation operators satisfy deformed commutation relations and the Fock space of states is constructed in this formalism in terms of basic numbers familiar from the theory of quantum groups. Expressions for the Hamiltonian and momentum arising from deformed Heisenberg relations are obtained and their consequences investigated. The energy momentum properties of the vacuum state are studied. The commutation relation for the fields is shown to involve polarization sums more intricate than those encountered in standard quantum electrodynamics, thus requiring explicit representations of polarization vectors. The electric field commutation rules are investigated under simplifying assumptions of polarization states, and the commutator in the deformed theory in this case is shown to be reminiscent of the coordinate-momentum uncertainty relation in the theory of q-deformed quantum oscillators.
\end{abstract}

Electronic address: pswamy@siue.edu

August 2004

PACS 03.65.-w, 03.70.+k, 12.20.-m, 02.20.Uw 


\section{INTRODUCTION}

The task of quantizing transverse electromagnetic radiation is facilitated by first establishing that the system of pure radiation fields is equivalent to a set of harmonic oscillators. As is well-known, this is accomplished by demonstrating that the Hamiltonian of the radiation fields is of the same mathematical form as the Hamiltonian of a set of harmonic oscillators [1]. Other quantities such as the momentum of radiation fields can also be cast in this form. The quantum theory of radiation is then straightforward and follows the steps of quantizing the system of harmonic oscillators. In other words the fundamental premise of the quantum theory of radiation has to do with the equivalence of transverse radiation and a set of non-interacting harmonic oscillators.

On the other hand we have gained a great deal of insight into the theory of q-deformed harmonic oscillators from the wisdom of decades of investigation [2-4] of a theory of the system, built on a Fock space of states arising from modified commutation relations for the annihilation and creation operators. Deformed oscillators are thus realized as quantum groups. The physical meaning of such a deformation can be interpreted as a modification of the Planck constant [2]. In other words, the q-deformation can be regarded as a modification of the Planck constant just as quantum mechanics might be regarded as a deformation of the classical system.

We may accordingly pose the following question. If the standard quantum theory of transverse electromagnetic radiation is completely equivalent to a system of harmonic oscillators, what kind of transverse radiation fields would correspond to a system of q-deformed harmonic oscillators? The purpose of this work is to formulate such a theory of pure transverse electromagnetic radiation and display its salient properties. This work is certainly not yet a complete investigation of all aspects of the theory but rather a modest, preliminary study to highlight some of the prominent features. This paper is organized as follows. Sec. II is devoted to the formulation of the theory of electromagnetic radiation that would correspond to a set of q-deformed harmonic oscillators. This formalism employs basic numbers. Sec.III deals with the Hamiltonian of the system expressed in terms of annihilation and creation operators obeying modified commutation relations. We also describe the construction of Fock space of photon states. In Sec.IV we investigate the momentum of the radiation fields. We also study the properties of the vacuum state. Sec.V contains a useful representation of the polarization vectors needed for our investigation. The rationale for this is as follows. This formalism is more complicated than standard quantum electrodynamics and most investigations would require an explicit representation of the polarization vectors satisfying the usual properties. In Sec. VI, we study the commutation relations satisfied by radiation fields, specifically the commutation relations of the electric field components. We illustrate the main feature of deformation of field commutation relations by considering a simplistic example of specific polarization states. Sec.VII contains a summary and concluding remarks. 


\section{THE FORMALISM}

We seek a plane wave representation of potentials $\mathbf{A}$, the conjugate momentum $\mathbf{P}$ and fields $\mathbf{E}, \mathbf{B}$ representing pure transverse radiation which will be consistent with q-deformed oscillators. We begin with the fact that the Hamiltonian of the standard quantized radiation fields is described by

$$
H=\int d^{3} r\left\{2 \pi c^{2}|\mathbf{P}|^{2}+\frac{1}{8 \pi}(\nabla \times \mathbf{A})^{2}\right\}
$$

where the momentum conjugate to the potential is

$$
\mathbf{P}=\frac{1}{4 \pi}\left(\frac{1}{c} \frac{\partial \mathbf{A}}{\partial t}+\nabla \phi\right)
$$

The well-known standard analysis [1,5-10] leads to the Hamiltonian of the form

$$
H=\sum_{\mathbf{k}, \lambda}^{\prime}\left(4 \pi c^{2} p_{\mathbf{k}, \lambda}^{\dagger} p_{\mathbf{k}, \lambda}+\frac{k^{2}}{4 \pi} x_{\mathbf{k}, \lambda}^{\dagger} x_{\mathbf{k}, \lambda}\right),
$$

which is obtained from the expansions

$$
\mathbf{A}(\mathbf{r}, t)=\sum_{\mathbf{k}, \lambda}^{\prime}\left\{x_{\mathbf{k}, \lambda}(t) \mathbf{u}_{\mathbf{k}, \lambda}(\mathbf{r})+x_{\mathbf{k}, \lambda}^{\dagger}(t) \mathbf{u}_{\mathbf{k}, \lambda}^{*}(\mathbf{r})\right\}
$$

and

$$
\mathbf{P}(\mathbf{r}, t)=\sum_{\mathbf{k}, \lambda}^{\prime}\left\{p_{\mathbf{k}, \lambda}(t) \mathbf{u}_{\mathbf{k}, \lambda}(\mathbf{r})+p_{\mathbf{k}, \lambda}^{\dagger}(t) \mathbf{u}_{\mathbf{k}, \lambda}^{*}(\mathbf{r})\right\} .
$$

Here the sum over $\lambda=1,2$ corresponds to the two transverse polarization degrees of freedom. The foundations of the quantum theory of radiation formulated a long time ago [1] need to be generalized. We shall adhere to the notation employed in [8], which includes many of the nuances of the analysis. The prime accompanying the expansions above indicates that the sum is over only half the $\mathbf{k}$-space i.e., positive $\mathbf{k}$ direction: this avoids duplication of $\mathbf{u}_{-\mathbf{k}, \lambda}$ in $\mathbf{u}_{\mathbf{k}, \lambda}^{*}$. At the end of the calculation [8], we shall be able to replace it by the ordinary sum over all of $\mathbf{k}$-space. The dynamical variables $x_{\mathbf{k}, \lambda}$ and $p_{\mathbf{k}, \lambda}$, the coordinates and momenta, are formal objects introduced so that the form of the Hamiltonian, Eq.(3), is that of harmonic oscillators. The plane wave representation is necessary to express the potentials and fields in a complete orthonormal set of plane waves according to

$$
\mathbf{u}_{\mathbf{k}, \lambda}(\mathbf{r})=L^{-3 / 2} \varepsilon_{\mathbf{k}, \lambda} e^{i \mathbf{k} \cdot \mathbf{r}} .
$$

Thus for each momentum $\mathbf{k}$ and polarization $\lambda=1,2$, the plane wave is constructed and employed in the expansions in Eqs.(3-5) and $\varepsilon_{\mathbf{k}, \lambda}$ represents the polarization vector describing transverse radiation for a given momentum and polarization direction.

The objects $x_{\mathbf{k}, \lambda} \mathbf{u}_{\mathbf{k}, \lambda}$ and $p_{\mathbf{k}, \lambda} \mathbf{u}_{\mathbf{k}, \lambda}$ in Eqs. $(4,5)$ can be expressed as linear combinations of plane waves $e^{i(\mathbf{k} \cdot \mathbf{r} \mp k c t)}$ with coefficient operators $a_{\mathbf{k}, \lambda}$ and $a_{\mathbf{k}, \lambda}^{\prime \dagger}$ in the undeformed theory. In 
the deformed theory, however, these annihilation and creation operators play subtler roles, as we shall see.

Accordingly, we shall now proceed to introduce the basic premises of the formulation that will lead to the realization of transverse radiation as q-deformed harmonic oscillators.

1. First, the canonical commutation relations satisfied by the dynamical variables $x_{\mathbf{k}, \lambda}$ and $p_{\mathbf{k}, \lambda}$ in standard electrodynamics are

$$
\left[x_{\mathbf{k}, \lambda}, p_{\mathbf{k}^{\prime}, \lambda^{\prime}}^{\dagger}\right]=\left[x_{\mathbf{k}, \lambda}^{\dagger}, p_{\mathbf{k}^{\prime}, \lambda^{\prime}}\right]=i \hbar \delta_{\mathbf{k k}^{\prime}} \delta_{\lambda \lambda^{\prime}}
$$

which must now be modified by the following commutation relations valid for the q-deformed system:

$$
\left[x_{\mathbf{k}, \lambda}, p_{\mathbf{k}^{\prime}, \lambda^{\prime}}^{\dagger}\right]=\left[x_{\mathbf{k}, \lambda}^{\dagger}, p_{\mathbf{k}^{\prime}, \lambda^{\prime}}\right]=i \hbar \delta_{\mathbf{k k}^{\prime}} \delta_{\lambda \lambda^{\prime}}\left(\left[N_{\mathbf{k}, \lambda}+1\right]-\left[N_{\mathbf{k}, \lambda}\right]\right)
$$

where $[a, b]=a b-b a$. The commutator itself thus remains unmodified and the right hand side displays the effect of deformation. The bracket numbers on the right hand side here denote the basic numbers defined by

$$
[x]=\frac{q^{x}-q^{-x}}{q-q^{-1}}
$$

where $q$ is the parameter (base) signifying deformation. The bracket number or the basic number $[N]$ is not the number operator but $N$ is. In this formulation [11] which is symmetric under $q \longrightarrow q^{-1}$, it suffices to restrict its range to $0<q<1$ with no loss of generality. There are many useful properties of bracket numbers known in the literature, such as the following representation:

$$
[x]=\frac{\sinh x \gamma}{\sinh \gamma}, \quad q=e^{\gamma} .
$$

2. The time dependence of operators is governed by Heisenberg equations of motion,

$$
i \hbar \frac{\partial}{\partial t} x_{\mathbf{k}, \lambda}(t)=\left[x_{\mathbf{k}, \lambda}(t), H\right]=4 \pi i \hbar c^{2} p_{\mathbf{k}, \lambda}
$$

and

$$
i \hbar \frac{\partial}{\partial t} p_{\mathbf{k}, \lambda}(t)=\left[p_{\mathbf{k}, \lambda}(t), H\right]=-\frac{i \hbar k^{2}}{4 \pi} x_{\mathbf{k}, \lambda}
$$

where $k=|\mathbf{k}|$. There is thus no deformation introduced in the time dependence. We could have introduced deformation of the equations of motion governing time dependence here by employing the Jackson derivative, playing an important role leading to q-calculus [12], which is known to preserve the whole structure of thermostatistics. However, we shall refrain from such a generalization here.

3. For the deformed oscillators, $\left[N_{\mathbf{k}, \lambda}\right]$ is not the number operator; instead the bracket numbers and the annihilation and creation operators are related by

$$
\left[N_{\mathbf{k}, \lambda}\right]=\frac{k}{2 \pi \hbar c} a_{\mathbf{k}, \lambda}^{\dagger} a_{\mathbf{k}, \lambda} ; \quad\left[N_{\mathbf{k}, \lambda}+1\right]=\frac{k}{2 \pi \hbar c} a_{\mathbf{k}, \lambda} a_{\mathbf{k}, \lambda}^{\dagger}
$$


Our analysis below will enable us to unambiguously establish the above identification.

We are now ready to derive the consequences of the basic premises introduced above. Eqs.(11,12) immediately lead to

$$
\frac{\partial^{2}}{\partial t^{2}} x_{k, \lambda}(t)=-k^{2} c^{2} x_{k, \lambda}(t)
$$

and a similar differential equation for $p_{k, \lambda}(t)$. Consequently, we obtain the solutions in the form of the linear combination

$$
x_{\mathbf{k}, \lambda}(t)=a_{\mathbf{k}, \lambda} e^{-i k c t}+a_{\mathbf{k}, \lambda}^{\prime \dagger} e^{i k c t}
$$

and

$$
p_{\mathbf{k}, \lambda}(t)=-\frac{i k}{4 \pi c}\left\{a_{\mathbf{k}, \lambda} e^{i k c t}-a_{\mathbf{k}, \lambda}^{\prime \dagger} e^{i k c t}\right\}
$$

just as in the undeformed theory [8]. At the end of the calculation, we shall be able to identify $a_{\mathbf{k}, \lambda}^{\prime}$ with $a_{-\mathbf{k}, \lambda}$. Inverting Eqs. $(15,16)$, we obtain the relations

$$
\begin{aligned}
& a_{\mathbf{k}, \lambda}=\frac{1}{2}\left\{x_{\mathbf{k}, \lambda}+\frac{4 \pi i c}{k} p_{\mathbf{k}, \lambda}\right\} e^{i k c t}, \\
& a_{\mathbf{k}, \lambda}^{\prime \dagger}=\frac{1}{2}\left\{x_{\mathbf{k}, \lambda}-\frac{4 \pi i c}{k} p_{\mathbf{k}, \lambda}\right\} e^{-i k c t},
\end{aligned}
$$

and taking the hermitian conjugate leads to the further relations

$$
\begin{aligned}
& a_{\mathbf{k}, \lambda}^{\dagger}=\frac{1}{2}\left\{x_{\mathbf{k}, \lambda}^{\dagger}-\frac{4 \pi i c}{k} p_{\mathbf{k}, \lambda}^{\dagger}\right\} e^{-i k c t}, \\
& a_{\mathbf{k}, \lambda}^{\prime}=\frac{1}{2}\left\{x_{\mathbf{k}, \lambda}^{\dagger}+\frac{4 \pi i c}{k} p_{\mathbf{k}, \lambda}^{\dagger}\right\} e^{i k c t}
\end{aligned}
$$

We can now evaluate the commutator, and after some algebra, obtain

$$
\left[a_{\mathbf{k}, \lambda}, a_{\mathbf{k}^{\prime}, \lambda^{\prime}}^{\dagger}\right]=\frac{2 \pi \hbar c}{k} \delta_{\mathbf{k k}^{\prime}} \delta_{\lambda \lambda^{\prime}}\left(\left[N_{\mathbf{k}, \lambda}+1\right]-\left[N_{\mathbf{k}, \lambda}\right]\right)
$$

upon utilizing Eq.(8). In the limit $q \rightarrow 1$ this goes over to the standard commutation relation since $\left[N_{k, \lambda}+1\right]-\left[N_{k, \lambda}\right] \rightarrow 1$ for all $\mathbf{k}, \lambda$. Similarly, we obtain from the second pair of equations,

$$
\left[a_{\mathbf{k}, \lambda}^{\prime}, a_{\mathbf{k}^{\prime}, \lambda^{\prime}}^{\prime \dagger}\right]=\frac{2 \pi \hbar c}{k} \delta_{\mathbf{k k}^{\prime}} \delta_{\lambda \lambda^{\prime}}\left(\left[N_{\mathbf{k}, \lambda}^{\prime}+1\right]-\left[N_{\mathbf{k}, \lambda}^{\prime}\right]\right)
$$

We observe that the above equations evidently confirm the relations contained in Eq.(13). 


\section{THE HAMILTONIAN}

The Hamiltonian given by Eq.(3), together with (8) describes q-deformed oscillators. We can evaluate it in terms of the creation and annihilation operators given by Eqs. $(17,18)$ and express the result as

$$
H=\sum_{\mathbf{k}, \lambda}^{\prime} \frac{k^{2}}{2 \pi}\left(a_{\mathbf{k}, \lambda} a_{\mathbf{k}, \lambda}^{\dagger}+a_{\mathbf{k}, \lambda}^{\dagger} a_{\mathbf{k}, \lambda}\right) .
$$

where the summation is over half the $\mathbf{k}$-space (positive $\mathbf{k}$ direction). Utilizing Eq.(13), the Hamiltonian can be cast in the form

$$
H=\sum_{\mathbf{k}, \lambda}^{\prime} \hbar c k\left(\left[N_{\mathbf{k}, \lambda}+1\right]+\left[N_{\mathbf{k}, \lambda}^{\prime}\right]\right) .
$$

Identifying $N_{\mathbf{k}, \lambda}^{\prime}$ in terms of negative $\mathbf{k}$ direction, we may now extend the sum over all of $\mathbf{k}$-space, thus removing the prime in the sum [8], and obtain the result

$$
H=\sum_{\mathbf{k}, \lambda} \frac{1}{2} \hbar c k\left(\left[N_{\mathbf{k}, \lambda}+1\right]+\left[N_{\mathbf{k}, \lambda}\right]\right)
$$

where the bracket numbers appear instead of the number operator for $q \neq 1$. This reduces to the standard result $\sum \hbar c k\left(N_{\mathbf{k}, \lambda}+\frac{1}{2}\right)$ in the limit $q \rightarrow 1$ of of the undeformed theory. Consequently, the result in Eq.(23) includes the zero point energy.

Finally, we may collect all the commutation relations in the form

$$
\begin{aligned}
& {\left[x_{\mathbf{k}, \lambda}, p_{\mathbf{k}, \lambda}^{\dagger}\right]=\left[x_{\mathbf{k}, \lambda}^{\dagger}, p_{\mathbf{k}, \lambda}\right]=i \hbar\left(\left[N_{\mathbf{k}, \lambda}+1\right]-\left[N_{\mathbf{k}, \lambda}\right]\right)} \\
& {\left[x_{\mathbf{k}, \lambda}^{\dagger}, p_{\mathbf{k}, \lambda}\right]=\left[x_{\mathbf{k}, \lambda}, p_{\mathbf{k}, \lambda}^{\dagger}\right]=i \hbar\left(\left[N_{\mathbf{k}, \lambda}^{\prime}+1\right]-\left[N_{\mathbf{k}, \lambda}\right]\right)}
\end{aligned}
$$

where, as before, the prime on $N_{\mathbf{k}, \lambda}$ can be identified with negative directions of $\mathbf{k}$.

It is important to observe that the deformed Heisenberg commutation relations for every $\mathbf{k}$ and $\lambda$ as in Eq.(8) directly lead to the identification in Eq.(13). Our analysis further shows that this leads to the Hamiltonian, Eq.(23), expressed in terms of the basic numbers, which are again essential in describing the deformed oscillators. We thus conclude that the Hamiltonian of transverse electromagnetic radiation, realized as deformed oscillators, is a direct consequence of the following ingredients of the theory: the q-deformed Heisenberg commutation relations for the dynamical variables $x_{\mathbf{k}, \lambda}, p_{\mathbf{k}, \lambda}$; the expressions for $a_{\mathbf{k}, \lambda}, a_{\mathbf{k}, \lambda}^{\dagger}$ in terms of the dynamical variables $x_{\mathbf{k}, \lambda}, p_{\mathbf{k}, \lambda}$; the time dependence given by the Heisenberg equations of motion.

We may arrive at the result, Eq.(23) directly from the expression for the energy of radiation fields, Eq.(1), as an instructive exercise; or equivalently from

$$
H=\frac{1}{8 \pi} \int d^{3} r\left(|E|^{2}+|B|^{2}\right)
$$

It suffices to consider the contribution from the electric field alone since that would be half the total. We then determine after a straightforward calculation: 


$$
\begin{aligned}
H= & \frac{1}{8 \pi} \int d^{3} r E^{i}(\mathbf{r}, t) E^{i}(\mathbf{r}, t) \\
= & \frac{1}{8 \pi} \int d^{3} r L^{-3} \sum_{\mathbf{k}, \lambda}^{\prime} \sum_{\mathbf{k}^{\prime}, \lambda^{\prime}}^{\prime}\left(k k^{\prime}\right) \varepsilon_{\mathbf{k}, \lambda}^{i} \varepsilon_{\mathbf{k}^{\prime}, \lambda^{\prime}}^{i} \\
& \times\left\{a_{\mathbf{k}, \lambda} e^{i(\mathbf{k} \cdot \mathbf{r}-k c t)}-a_{\mathbf{k}, \lambda}^{\dagger} e^{-i(\mathbf{k} \cdot \mathbf{r}-k c t)}\right\} \\
& \times\left\{a_{\mathbf{k}^{\prime}, \lambda^{\prime}}^{\dagger} e^{-i\left(\mathbf{k}^{\prime} \cdot \mathbf{r}-k^{\prime} c t\right)}-a_{\mathbf{k}^{\prime}, \lambda^{\prime}} e^{i\left(\mathbf{k}^{\prime} \cdot \mathbf{r}-k^{\prime} c t\right)}\right\} \\
= & \frac{1}{8 \pi} \sum_{\mathbf{k}, \lambda} k^{2}\left(a_{\mathbf{k}, \lambda} a_{\mathbf{k}, \lambda}^{\dagger}+a_{\mathbf{k}, \lambda}^{\dagger} a_{\mathbf{k}, \lambda}\right) .
\end{aligned}
$$

Hence we obtain the desired result

$$
H=\frac{1}{8 \pi} \int d^{3} r|E|^{2}=\sum_{\mathbf{k}, \lambda} \frac{1}{4} \hbar c k\left(\left[N_{\mathbf{k}, \lambda}+1\right]+\left[N_{\mathbf{k}, \lambda}\right]\right),
$$

where the sum is now over all of $\mathbf{k}$ space.

We shall now complete this section by discussing the Fock space of the deformed harmonic oscillators. We begin by observing that while the commutation relation such as in Eq.(19) is most convenient and natural, we can easily derive an alternative form. From Eq.(13), we immediately derive

$$
a_{\mathbf{k}, \lambda} a_{\mathbf{k}, \lambda}^{\dagger}-q a_{\mathbf{k}, \lambda}^{\dagger} a_{\mathbf{k}, \lambda}=q^{-N}
$$

which is the preferred form standard in the literature. We introduce the Fock states $\left|n_{\mathbf{k}, \lambda}\right\rangle$ as follows. By analyzing the action on this state by operators $a_{\mathbf{k}, \lambda}$ and $a_{\mathbf{k}, \lambda}^{\dagger}$ we find

$$
a_{\mathbf{k}, \lambda}^{\dagger}\left|n_{\mathbf{k}, \lambda}\right\rangle=\sqrt{\left[n_{\mathbf{k}, \lambda}+1\right]}\left|n_{\mathbf{k}, \lambda}\right\rangle ; \quad a_{\mathbf{k}, \lambda}\left|n_{\mathbf{k}, \lambda}\right\rangle=\sqrt{\left[n_{\mathbf{k}, \lambda}\right]}\left|n_{\mathbf{k}, \lambda}-1\right\rangle .
$$

Consequently we obtain, for any $\mathbf{k}, \lambda$,

$$
\left|n_{\mathbf{k}, \lambda}\right\rangle=\frac{\left(a_{\mathbf{k}, \lambda}^{\dagger}\right)^{n_{\mathbf{k}, \lambda}}}{\sqrt{\left[n_{\mathbf{k}, \lambda}\right] !}}|0\rangle
$$

where

$$
[r] !=[r][r-1] \cdots 1
$$

Accordingly we may build the general Fock state by

$$
\left|n_{\mathbf{k}_{1}, \lambda_{1}}, n_{\mathbf{k}_{2}, \lambda_{2}} \cdots\right\rangle=\left(\prod_{s} \frac{\left(a_{s}^{\dagger}\right)^{n_{s}}}{\sqrt{\left[n_{s}\right] !}}\right)|0\rangle
$$

where the abbreviation $s$ denotes $\mathbf{k}_{s}, \lambda_{s}$. 


\section{MOMENTUM OF RADIATION}

Starting from the definition of the momentum of the radiation fields

$$
\mathbf{G}=\frac{1}{4 \pi} \int d^{3} r(\mathbf{E} \times \mathbf{B})
$$

we may express it in terms of the dynamical variables as

$$
\mathbf{G}=i \sum_{\mathbf{k}, \lambda}^{\prime} \mathbf{k}\left(p_{\mathbf{k}, \lambda} x_{\mathbf{k}, \lambda}^{\dagger}-p_{\mathbf{k}, \lambda}^{\dagger} x_{\mathbf{k}, \lambda}\right)
$$

We shall now utilize Eqs. $(15,16)$ in order to express the momentum in terms of the creation and annihilation operators. Noting that $\left[a_{\mathbf{k}, \lambda}, a_{\mathbf{k}, \lambda}\right]=\left[a_{\mathbf{k}, \lambda}^{\dagger}, a_{\mathbf{k}, \lambda}^{\dagger}\right]=0$ and making use of the commutation relations, Eq. $(19,20)$, we obtain

$$
\mathbf{G}=\frac{1}{4 \pi c} \sum_{\mathbf{k}, \lambda}^{\prime} k \mathbf{k}\left\{\left(a_{\mathbf{k}, \lambda} a_{\mathbf{k}, \lambda}^{\dagger}+a_{\mathbf{k}, \lambda}^{\dagger} a_{\mathbf{k}, \lambda}\right)-\left(a_{\mathbf{k}, \lambda}^{\prime} a_{\mathbf{k}, \lambda}^{\prime \dagger}+a_{\mathbf{k}, \lambda}^{\prime \dagger} a_{\mathbf{k}, \lambda}^{\prime}\right)\right\}
$$

which can be readily expressed in the form

$$
\mathbf{G}=\frac{1}{2} \sum_{\mathbf{k}, \lambda}^{\prime} \hbar \mathbf{k}\left\{\left[N_{\mathbf{k}, \lambda}+1\right]+\left[N_{\mathbf{k}, \lambda}\right]-\left(\left[N_{\mathbf{k}, \lambda}^{\prime}+1\right]+\left[N_{\mathbf{k}, \lambda}^{\prime}\right]\right)\right\} .
$$

First, it is instructive study the limit $q \rightarrow 1$ in the undeformed theory. In this case the bracket numbers become ordinary numbers and the terms containing unity cancel. We can then express it in terms of the sum over the full $\mathbf{k}$-space in this case and obtain the familiar, standard result,

$$
\mathbf{G}=\sum_{\mathbf{k}, \lambda} \hbar \mathbf{k} N_{\mathbf{k}, \lambda}
$$

It is important to note that the zero point contribution cancels out and the sum above is over all of $\mathbf{k}$. Now returning to the case of $q \neq 1$, and identifying $N_{\mathbf{k}, \lambda}^{\prime}$ as belonging to negative $\mathbf{k}$, proceeding as before, we obtain the result

$$
\left.\mathbf{G}=\frac{1}{2} \sum_{\mathbf{k}, \lambda} \hbar \mathbf{k}\left(\left[N_{\mathbf{k}, \lambda}+1\right]+\left[N_{\mathbf{k}, \lambda}\right]\right)\right\} .
$$

Hence we conclude that when $q \neq 1$, the zero point contribution does not trivially vanish. On the other hand, by comparing with the Hamiltonian, we find that the operator energymomentum relation $H=c|G|$ is satisfied. Consequently, the expression for momentum of radiation is given by the two different expressions, Eqs. $(37,38)$ for $q=1$ and $q \neq 1$ and it would be misleading to take the limit directly in Eq.(38). It is interesting to note that in the undeformed case of $q=1$, the limit of Eq.(38) appears to be in agreement with the result in [5]. However it must be stressed that the above results are correct only in their respective domain. 
In view of the discussion above, it may be worthwhile to inspect the results more closely in order to remove any ambiguity. Eq.(36), may be alternatively re-expressed as

$$
\mathbf{G}=\frac{1}{2} \sum_{\mathbf{k}, \lambda}^{\prime} \hbar \mathbf{k}\left\{q\left[N_{\mathbf{k}, \lambda}\right]+q^{-N_{\mathbf{k}, \lambda}}+\left[N_{\mathbf{k}, \lambda}\right]-\left(q\left[N_{\mathbf{k}, \lambda}^{\prime}\right]+q^{-N_{\mathbf{k}, \lambda}^{\prime}}+\left[N_{\mathbf{k}, \lambda}^{\prime}\right]\right)\right\} .
$$

since $[N+1]=q[N]+q^{-N}$. The numbers $N^{\prime}$ are identified with negative momentum values. Finally we can combine into a sum over the full $\mathbf{k}$-space and thus obtain

$$
\mathbf{G}=\frac{1}{2} \sum_{\mathbf{k}, \lambda} \hbar \mathbf{k}\left((q+1)[N]+q^{-N}\right),
$$

where the sum is without the prime, thus confirming the previous result in Eq.(38). Accordingly we may summarize our results as follows:

$$
\mathbf{G}=\left\{\begin{array}{cc}
\sum_{\mathbf{k}, \lambda} \hbar \mathbf{k} N_{\mathbf{k}, \lambda}, & q=1, \\
\sum_{\mathbf{k}, \lambda} \frac{1}{2} \hbar \mathbf{k}\left(\left[N_{\mathbf{k}, \lambda}+1\right]+\left[N_{\mathbf{k}, \lambda}\right]\right), & q \neq 1
\end{array}\right.
$$

and

$$
H=\left\{\begin{array}{cc}
\sum_{\mathbf{k}, \lambda} \hbar c k\left(N_{\mathbf{k}, \lambda}+\frac{1}{2}\right), & q=1 \\
\frac{1}{2} \sum_{\mathbf{k}, \lambda} \hbar c k\left(\left[N_{\mathbf{k}, \lambda}+1\right]+\left[N_{\mathbf{k}, \lambda}\right]\right), & q \neq 1
\end{array}\right.
$$

We conclude this section by discussing the energy and momentum eigenvalues for the vacuum state, which seems to be of considerable importance, thus providing an explicit clarification and reiteration of the above discussion. First we observe that

$$
N|0\rangle=0, \quad[N]|0\rangle=0
$$

The latter result follows from the representation $[N]=\sinh N \gamma / \sinh \gamma$. In a similar manner, we observe that

$$
(N+1)|0\rangle=|0\rangle, \quad[N+1]|0\rangle=|0\rangle .
$$

As a result, we are now able to directly examine Eqs.(41,42) and conclude that for $q=1$, the momentum of the vacuum state vanishes whereas it has zero point energy; on the other hand, in the undeformed case, $q \neq 1$, both the momentum and energy have zero point values.

\section{POLARIZATION VECTORS}

In order to proceed with further investigations, it is necessary to study the polarization vectors describing transverse electromagnetic radiation in the deformed theory, although this is a straightforward matter in ordinary electrodynamics. We know that the polarization vectors $\varepsilon_{\lambda}^{i}$ are defined by orthogonal unit vectors [1,5-10] satisfying the property of orthonormality 


$$
\sum_{i=1}^{3} \varepsilon_{\lambda}^{i} \varepsilon_{\lambda^{\prime}}^{i}=\delta_{\lambda \lambda^{\prime}}
$$

where the space and polarization indices are $i=1,2,3$ and $\lambda=1,2$; and the completeness relation

$$
\sum_{\lambda=1,2} \varepsilon_{\lambda}^{i} \varepsilon_{\lambda}^{j}=\delta_{i j}-\frac{k_{i} k_{j}}{k^{2}}
$$

The completeness property above is rather obvious since the unit vectors would ordinarily satisfy

$$
\sum_{\lambda=1}^{3} \varepsilon_{\lambda}^{i} \varepsilon_{\lambda}^{j}=\delta_{i j}
$$

if the polarization were to be summed over $\lambda=1,2,3$; we merely need to introduce $\varepsilon_{3}^{i}=$ $k_{i} /|k|$ and subtract the product $k_{i} k_{j} /|k|^{2}$ from the above to obtain the sum over $\lambda=1,2$, leading to Eq.(46).

In order to perform many calculations involving the sum over polarizations in the deformed theory, such as the evaluation of $\sum_{\lambda} \varepsilon_{\lambda}^{i} \mathcal{O}_{\lambda} \varepsilon_{\lambda}^{j}$, we need an explicit representation of the polarization vectors satisfying the above properties and for this purpose we proceed as follows.

Given an arbitrary vector $\mathbf{A}$, the transverse polarization vectors are easily constructed by the prescription

$$
\varepsilon_{1}=\alpha\left(\mathbf{A}-\frac{\mathbf{k}(k \cdot A)}{k^{2}}\right), \quad \varepsilon_{2}=\frac{\mathbf{k} \times \varepsilon_{1}}{|k|}=\alpha \frac{\mathbf{k} \times \mathbf{A}}{|k|}
$$

so that, together with $\hat{\mathbf{k}}$, they form a right-handed triad of unit vectors. We determine the normalization to be

$$
\alpha^{2}=\frac{k^{2}}{k^{2} A^{2}-(k \cdot A)^{2}},
$$

which still provides a generous class of vectors $\mathbf{A}$ for our purpose. It is easily verified that they satisfy the orthonormality property, Eq.(45). Proceeding to confirm the completeness property we obtain

$$
\left.\varepsilon_{\lambda}^{i} \varepsilon_{\lambda}^{j}=\alpha^{2}\left(A_{i}-\frac{k_{i}(k \cdot A)}{k^{2}}\right)\left(A_{j}-\frac{k_{j}(k \cdot A)}{k^{2}}\right)+\frac{\alpha^{2}}{k^{2}}(\mathbf{k} \times \mathbf{A})_{i} \mathbf{k} \times \mathbf{A}\right)_{j} .
$$

This is difficult to analyze for arbitrary $\mathbf{A}$ and hence it is prudent to choose a convenient vector.

Choice 1: if we choose $\mathbf{A}=\hat{\mathbf{x}}$, we can immediately verify the orthonormality property, Eq.(45). We also find

$$
\varepsilon_{1}^{i} \varepsilon_{1}^{j}+\varepsilon_{2}^{i} \varepsilon_{2}^{j}=\alpha^{2}\left(\hat{\mathbf{x}}-\frac{\mathbf{k} k_{1}}{k^{2}}\right)_{i}\left(\hat{\mathbf{x}}-\frac{\mathbf{k} k_{1}}{k^{2}}\right)_{j}+\frac{\alpha^{2}}{k^{2}}\left(k_{3} \hat{\mathbf{y}}-k_{2} \hat{\mathbf{z}}\right)_{i}\left(k_{3} \hat{\mathbf{y}}-k_{2} \hat{\mathbf{z}}\right)_{j} .
$$


Explicitly, we can verify the following results

$$
\begin{aligned}
& \varepsilon_{1}^{1} \varepsilon_{1}^{1}+\varepsilon_{2}^{1} \varepsilon_{2}^{1}=1-\frac{k_{1}^{2}}{k^{2}}, \quad \varepsilon_{1}^{2} \varepsilon_{1}^{2}+\varepsilon_{2}^{2} \varepsilon_{2}^{2}=1-\frac{k_{2}^{2}}{k^{2}}, \\
& \varepsilon_{1}^{1} \varepsilon_{1}^{2}+\varepsilon_{2}^{1} \varepsilon_{2}^{2}=-\frac{k_{1} k_{2}}{k^{2}}, \quad \varepsilon_{1}^{3} \varepsilon_{1}^{3}+\varepsilon_{2}^{3} \varepsilon_{2}^{3}=1-\frac{k_{3}^{2}}{k^{2}}, \text { etc. }
\end{aligned}
$$

and thus verify the completeness relation, Eq.(46). We shall have occasion to utilize the above result in the next section.

There could be other choices. We find $\mathbf{A}=\hat{\mathbf{k}}$ is not desirable since it does not produce a non-zero polarization vector.

Choice 2: we may take $\mathbf{A}=(\hat{\mathbf{n}} \times \hat{\mathbf{k}})$ as our second choice, where $\hat{\mathbf{n}}$ is an arbitrary unit vector. We thus have, in this case,

$$
\varepsilon_{1}=\beta(\hat{\mathbf{n}} \times \hat{\mathbf{k}}), \quad \varepsilon_{2}=\beta(\hat{\mathbf{n}}-\hat{\mathbf{k}}(\hat{\mathbf{n}} \cdot \hat{\mathbf{k}})) .
$$

The normalization constant must now be

$$
\beta^{2}=\frac{k^{2}}{k^{2}-(n \cdot k)^{2}} .
$$

This satisfies the orthonormality property. If we specialize to the case $\hat{\mathbf{n}}=\hat{\mathbf{x}}$, we further obtain the result

$$
\varepsilon_{1}^{i} \varepsilon_{1}^{j}+\varepsilon_{2}^{i} \varepsilon_{2}^{j}=\frac{\beta^{2}}{k^{2}}\left(k_{2} \hat{\mathbf{z}}-k_{3} \hat{\mathbf{y}}\right)_{i}\left(k_{2} \hat{\mathbf{z}}-k_{3} \hat{\mathbf{y}}\right)_{j}+\beta^{2}\left(\hat{\mathbf{x}}-\frac{\mathbf{k} k_{1}}{k^{2}}\right)_{i}\left(\hat{\mathbf{x}}-\frac{\mathbf{k} k_{1}}{k^{2}}\right)_{j} .
$$

Calculating with the various components, we can again verify the results stated in Eq.(52) and thus verify the completeness property. We shall also utilize this result in the next section.

\section{CANONICAL COMMUTATION RELATIONS FOR FIELDS}

We shall now investigate the field commutation relations corresponding to the q-deformed oscillators. In order to highlight the prominent features, it suffices to consider the commu-

tation relation of a typical pair such as that of the electric field components. Employing the expansions

$$
E_{i}(\mathbf{r}, t)=L^{-3 / 2} \sum_{\mathbf{k}, \lambda}(i k) \varepsilon_{\mathbf{k}, \lambda}^{i}\left\{a_{\mathbf{k}, \lambda} e^{i(k \cdot r-k c t)}-a_{\mathbf{k}, \lambda}^{\dagger} e^{-i(k \cdot r-k c t)}\right\}
$$

and

$$
E_{j}\left(\mathbf{r}^{\prime}, t^{\prime}\right)=L^{-3 / 2} \sum_{\mathbf{k}^{\prime}, \lambda^{\prime}}\left(i k^{\prime}\right) \varepsilon_{\mathbf{k}^{\prime}, \lambda^{\prime}}^{j}\left\{a_{\mathbf{k}^{\prime}, \lambda^{\prime}} e^{i\left(k^{\prime} \cdot r^{\prime}-k^{\prime} c t^{\prime}\right)}-a_{\mathbf{k}^{\prime}, \lambda^{\prime}}^{\dagger} e^{-i\left(k^{\prime} \cdot r^{\prime}-k^{\prime} c t^{\prime}\right)}\right\}
$$

we derive the result

$$
\left[E_{i}(\mathbf{r}, t), E_{j}\left(\mathbf{r}^{\prime}, t^{\prime}\right)\right]=4 \pi i \hbar c L^{-3} \sum_{\mathbf{k}, \lambda} k \varepsilon_{\lambda}^{i} \varepsilon_{\lambda}^{j} \sin (\mathbf{k} \cdot \boldsymbol{\rho}-k c \tau)\left(\left[N_{\mathbf{k}, \lambda}+1\right]-\left[N_{\mathbf{k}, \lambda}\right]\right) .
$$


where $\boldsymbol{\rho}=\mathbf{r}-\mathbf{r}^{\prime}, \tau=t-t^{\prime}$. It is readily verified that, for the undeformed case [8], this reduces to :

$$
\lim _{q \rightarrow 1}\left[E_{i}(\mathbf{r}, t), E_{j}\left(\mathbf{r}^{\prime}, t^{\prime}\right)\right]=L^{-3}(4 \pi i \hbar c) \sum_{\mathbf{k}, \lambda} k \varepsilon_{\lambda}^{i} \varepsilon_{\lambda}^{j} \sin (\mathbf{k} \cdot \boldsymbol{\rho}-k c \tau) .
$$

To determine this when $q \neq 1$, we need to evaluate the polarization sum

$$
\sum_{\lambda} \varepsilon_{\lambda}^{i} \varepsilon_{\lambda}^{j}\left(\left[N_{\mathbf{k}, \lambda}+1\right]-\left[N_{\mathbf{k}, \lambda}\right]\right) .
$$

From Eq.(50), this turns out to be

$$
\sum_{\lambda} \alpha^{2}\left\{\left(A_{i}-\frac{k_{i}(k \cdot A)}{k^{2}}\right)\left(A_{j}-\frac{k_{j}(k \cdot A)}{k^{2}}\right)+\frac{1}{k^{2}}(\mathbf{k} \times \mathbf{A})_{i}(\mathbf{k} \times \mathbf{A})_{j}\right\}\left(\left[N_{\mathbf{k}, \lambda}+1\right]-\left[N_{\mathbf{k}, \lambda}\right]\right) .
$$

This is intractable due to the arbitrariness of $\mathbf{A}$ and the dependence of $[N]$ on $\mathbf{k}$ and $\lambda$. We shall consider the special choice of last section, the first choice, $\mathbf{A}=\hat{\mathbf{x}}$. Again, in order to study the nature of the deformation, we shall also consider only the components corresponding to $i=1, j=1$. In this case the polarization sum reduces to

$$
\left(1-\frac{k_{1}^{2}}{k^{2}}\right)\left(\left[N_{\mathbf{k}, 1}+1\right]-\left[N_{\mathbf{k}, 1}\right]\right)
$$

since only $\lambda=1$ survives. Replacing the sum by an integral, we thus determine the commutation relation to be

$$
\left[E_{1}(\mathbf{r}, t), E_{1}\left(\mathbf{r}^{\prime}, t^{\prime}\right)\right]=\frac{1}{(2 \pi)^{3}} \int d^{3} k \frac{1}{k}\left(\left[N_{\mathbf{k}, 1}+1\right]-\left[N_{\mathbf{k}, 1}\right]\right) \sin (\mathbf{k} \cdot \boldsymbol{\rho}-k c \tau) .
$$

Even after the simplifying choices made, the modified commutation relation is considerably different from the undeformed case. The deformation is the simplest when the number operator is independent of $\mathbf{k}$. In this case, we have

$$
\left(\left[N_{1}+1\right]-\left[N_{1}\right]\right)=\frac{\cosh \left(N_{1}+\frac{1}{2}\right) \gamma}{\cosh \frac{1}{2} \gamma},
$$

and this factor may be taken outside the integral. If we make the second choice as in Eq.(53), we obtain for the same pair of components,

$$
\left[E_{1}(\mathbf{r}, t), E_{1}\left(\mathbf{r}^{\prime}, t^{\prime}\right)\right]=\frac{1}{(2 \pi)^{3}} \int d^{3} k \frac{1}{k}\left(\left[N_{\mathbf{k}, 2}+1\right]-\left[N_{\mathbf{k}, 2}\right]\right) \sin (\mathbf{k} \cdot \boldsymbol{\rho}-k c \tau),
$$

since the $\lambda=2$ term survives. In such cases, and only under the special choices, the commutator is scaled by the factor above which is the same factor that appears in the fundamental commutation relation [2], the uncertainty relation of q-deformed oscillators :

$$
[x, p]=i \hbar([N+1]-[N])=\frac{\cosh \left(N+\frac{1}{2}\right) \gamma}{\cosh \gamma}
$$

More generally, the right hand side of Eqs. $(63,65)$ would be rather intricate due to the dependence on $\mathbf{k}$ and $\lambda$ and the derivative operations on the Green function and subsequent integration would be a formidable task. However, in the simple circumstance described above, the q-deformation is revealed by the factor $\left(\left[N_{1}+1\right]-\left[N_{1}\right]\right)$ or $\left(\left[N_{2}+1\right]-\left[N_{2}\right]\right)$. 


\section{CONCLUDING REMARKS}

We have investigated the formalism of quantized radiation fields corresponding to a set of q-deformed harmonic oscillators. This formalism is based on expressing the Hamiltonian of transverse radiation fields in terms of the dynamical variables, analogous to coordinates and momenta, which satisfy deformed Heisenberg uncertainty relations. The resulting quantum theory of transverse radiation is accordingly equivalent to q-deformed harmonic oscillators familiar from quantum group investigations. This formalism is technically complicated due to the vector nature of the radiation fields, with momentum vector and polarization vector appearing as degrees of freedom of the oscillators. Our goal has been to study the salient features of the theory such as the Hamiltonian, the momentum and canonical commutation relations for the electric field components without being daunted by the complications, rather than investigate all the nuances of the theory.

We have avoided using Jackson derivatives leading to a more general form of time dependence for the dynamical variables and concomitantly for the annihilation and creation operators. Although the details are not explicitly presented in this work, we have verified that the JD would lead to time dependence via q-exponential functions $E_{q}( \pm i k c t)$ instead of the ordinary exponential functions and the field commutation relations will be further complicated by dependence on time through q-sine functions. We have taken the approach requiring the simplest deformation.

The task of quantizing radiation in the manifestly covariant formulation is further complicated by the use of Lorentz gauge, requiring a four dimensional analysis as well as the full use of gauge invariance. It also requires the use of four component polarization vectors $[6,10]$. This is, however, unnecessary in the present investigation and we have succeeded in employing a simpler approach by working in the radiation gauge so that we restrict ourselves to transverse polarizations.

The complication of the vector nature of the fields is such that even with two polarization directions, many calculations would involve polarization sums including projection operators. This problem is not encountered in standard quantum electrodynamics. For this reason, we had to investigate and establish explicit representations for the polarization vectors which play important roles in the analysis.

We have shown how to construct the Fock space of states in this formalism. We have some interesting conclusions about the vacuum state in this formalism, especially the energy and momentum of the vacuum state.

Since the commutation relations for the field components are quite complicated, we are unable to obtain general results which are exact. We study only the electric field commutation relations. We are able to show the effect of q-deformation for the electric field commutation relations for different space and time points, as a simple factor containing a modified Planck constant, only under simplifying assumptions about polarization directions and the assumed lack of dependence of the number operator on photon momentum. It would certainly be worthwhile to explore this formalism further and obtain more general results. 


\section{REFERENCES}

[1] W.Heitler, The quantum theory of radiation, Oxford at the clarendon Press (1954) Third Edition.

[2] L.Biedenharn, J. Phys.A 22 (1989) L873.

[3] A. Macfarlane, J. Phys.A 22 (1989) 4581.

[4] P. Narayana Swamy, Int. J. Mod. Phys.B 10(1996) 683; A. Lavagno and P. Narayana Swamy, Phys. Rev. E 65 (2002) 036101 (contain a full list of references on the subject).

[5] V.B.Berestetskii, E.M.Lifshitz and L.P.Pitaevskii, Quantum Electrodynamics, Course of Theoretical Physics volume 4, Translated from the Russian, Pergamon Press, Oxford (1982) Second edition

[6] D.Lurie, Particles and Fields, Interscience Publishers, New York (1978).

[7] G.L.Trigg, Quantum Mechanics, D.Van Nostrand Co. Princeton (1964).

[8] L.I.Schiff, Quantum Mechanics McGraw-Hill Book Co. New York (1968), Third edition.

[9] J.D.Bjorken, S.D.Drell, Relativistic Quantum Fields, McGraw-Hill Book Co. (1965) New York.

[10] S.Gasiorowicz, Elementary Particle Physics John Wiley and Sons Inc. (1967) New York.

[11] H. Exton, q-Hypergeometric functions and applications, Ellis Horwood Limited (1983) Chichester; G. Gasper and M. Rahman, Basic hypergeometric series, Cambridge University Press (1990) Cambridge. It should be emphasized that, unlike in these references, the formulation that we have employed in this work is symmetric under $q \rightarrow q^{-1}$ and many of the well-established formulas need to be generalized.

[12] A.Lavagno and P. Narayana Swamy, Phys. Rev.E 61 (2000) 1218. 